\title{
Concentrated-mass cantilever enhances multiple harmonics in tapping-mode atomic force microscopy
}

\author{
Huiling Li, ${ }^{\text {a) }}$ Yan Chen, and Lanhong Dai \\ State Key Laboratory of Nonlinear Mechanics (LNM), Institute of Mechanics, Chinese Academy of Sciences, \\ Beijing 100190, People's Republic of China
}

(Received 3 March 2008; accepted 26 March 2008; published online 14 April 2008)

\begin{abstract}
The natural frequencies of a cantilever probe can be tuned with an attached concentrated mass to coincide with the higher harmonics generated in a tapping-mode atomic force microscopy by the nonlinear tip-sample interaction force. We provide a comprehensive map to guide the choice of the mass and the position of the attached particle in order to significantly enhance the higher harmonic signals containing information on the material properties. The first three eigenmodes can be simultaneously excited with only one carefully positioned particle of specific mass to enhance multiple harmonics. Accessing the interaction force qualitatively based on the high-sensitive harmonic signals combines the real-time material characterization with the imaging capability.

(C) 2008 American Institute of Physics. [DOI: 10.1063/1.2909535]
\end{abstract}

The tapping-mode atomic force microscopy ${ }^{1,2}$ is one of the most widely used high-resolution proximal probe techniques. In fact, the intermittent contact between the tip and the sample surface effectively eliminates the influence of the lateral adhesion and friction and, thus, allows the topography imaging of rather soft or fragile samples. The tip periodically tapping on the sample surface gives rise to a periodic pulselike tip-sample force, which generates high-frequency vibration components that are harmonics of the excitation frequency. ${ }^{3}$ The appearance of the higher harmonic signals serves as a sensitive index indicating the onset of such a nonlinear anharmonic interaction force. ${ }^{4}$ Thus, in an attempt to access the tip-sample interaction which can be quantitatively related to the material properties, the higher-order harmonic components in the vibration spectrum of the tapping cantilevers have attracted particular attention in the recent years. $^{5-10}$ A more detailed imaging with a better contrast which reveals the variation in material properties was obtained with the higher-harmonic resonance signal than that with the fundamental one ${ }^{11-13}$ while theoretical investigations and numerical simulations revealed that the high-order harmonic components contain the information on elastic properties of the sample surface. ${ }^{7,9,13}$ The high force sensitivity of higher harmonics or modes has been quantitatively examined by Lozano and Garcia. ${ }^{14}$ Unfortunately, the detection of higher harmonics with a reasonable signal-to-noise ratio is not always possible. ${ }^{3,12}$

While the cantilever is excited at or near its fundamental natural frequency to ensure a high-sensitive signal output of vibration amplitudes mainly corresponding to the fundamental eigenmode during the topography imaging, signal contributions from the higher harmonics of vibration amplitude are usually suppressed by the rapid decay of the frequency response curve of a continuum cantilever. ${ }^{15}$ In fact, the frequency response curve only exhibits high peaks near the natural frequencies corresponding to each of the vibration eigenmodes, as shown in Fig. 1. Thus, a higher harmonic signal with a frequency at an integer multiple of the funda-

a) Author to whom correspondence should be addressed. Electronic mail: lihl@lnm.imech.ac.cn. mental frequency seldom exactly coincides with these natural frequencies of the uniform cantilever, which may result in a rather small signal output that even falls below the effective noise level introduced by the detection electronics. Efforts have been made to enhance the higher harmonics, either by simultaneous exciting the cantilever at the first two natural frequencies, ${ }^{16}$ by driving the cantilever at a submultiple of the fundamental resonance frequency, ${ }^{17}$ or by exciting the torsional modes in a torsional harmonic cantilever. ${ }^{18}$ Instead of its excitation, the cantilever itself as a force sensor can also be optimized. A specialized harmonic cantilever with a special geometry shape precisely designed based on the finite element analysis has been invented by Sahin et al. ${ }^{15}$ to match the third natural frequency to the 16th harmonic, and the simultaneous excitation of the third eigenmode effectively increases the contrast in mapping elasticity variation across a surface. However, the lack of a comprehensive understanding of the influences of a rather complicated geometry shape which changes both the mass distribution and stiffness of the cantilever still prevents such harmonic cantilever from being precisely designed in a simple and flexible manner to meet the demand of practical applications.

In this letter, we propose a straightforward way to tune the typical cantilever having an uniform mass distribution along the length direction with an attached concentrated mass. In this way, the resonance peaks of the vibration spectrum of a original uniform cantilever can be tuned to exactly coincide with certain specific higher harmonics which we choose. The second and third eigemodes can be simultaneously excited together with the fundamental with only one carefully positioned particle of specific mass. The concentrated mass changes the vibration spectrum characteristics of the original uniform cantilever to enhance higher harmonic signals caused by the tip-sample interaction. The enhanced harmonic signals provide high-sensitive information to access the tip-sample interaction force which may be quantitatively related to material properties.

Attach a concentrated mass $m$ to the uniform cantilever at the location $x=x_{0}$, as shown in the inset of Fig. 1. Since the vibration amplitude $(\sim 100 \mathrm{~nm})$ is much less than the thickness of the cantilever $(\sim 1 \mu \mathrm{m})$, one-dimensional 


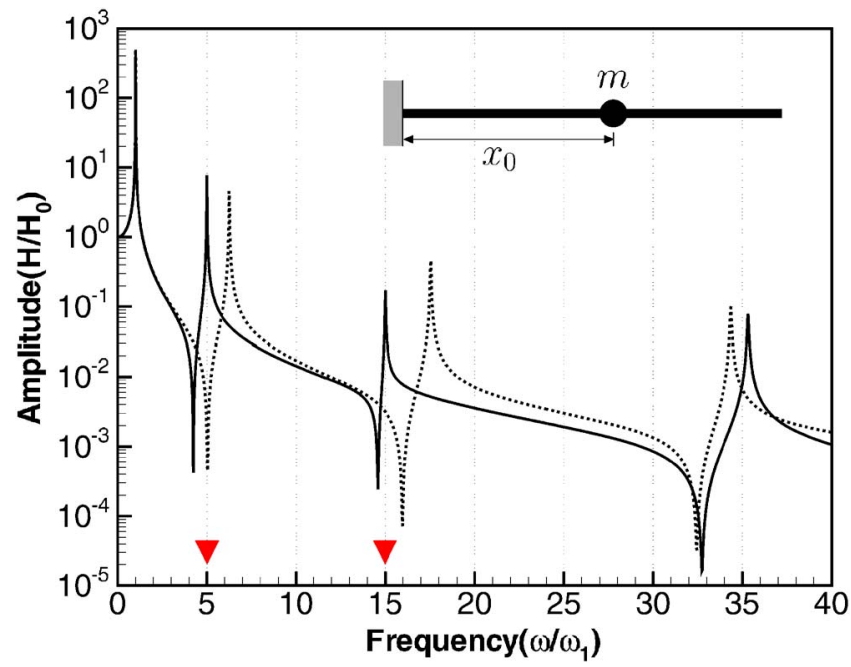

FIG. 1. (Color online) Frequency response curve of a harmonic cantilever with an attached concentrated mass (solid line) is compared with that of an uniform cantilever (dotted line). [The frequency $\omega$ and the vibration magnitude $H$ are normalized by the fundamental natural frequency $\omega_{1}$ and the amplitude $H_{0}$ at low frequency $(\omega \rightarrow 0)$, respectively. $\alpha=0.424, \xi_{0}=0.351$, the quality factor $Q=500$.] Arrows indicate the 5th and 15th harmonics of the fundamental frequency which are significantly enhanced by the second and third eigenmodes of the harmonic cantilever, respectively.

Euler-Bernouli equation for small deflection is used to investigate natural flexural vibrations of the concentrated-mass cantilever as

$$
E I \frac{\partial^{4} w(x, t)}{\partial x^{4}}+\left[\rho A+m \delta\left(x-x_{0}\right)\right] \frac{\partial^{2} w(x, t)}{\partial t^{2}}=0,
$$

where $w(x, t)$ is the time-dependent flexural displacement, $E$ is the Young's modulus, $I$ the moment of inertia, $\rho$ the mass density of the material, $A$ the cross-sectional area, and $\delta(x)$ is the Dirac's delta function. By writing $w(x, t)=Y(x) T(t)$, we are led to a boundary value problem of the nondimensional form

$$
\frac{d^{4} Y(\xi)}{d \xi^{4}}-\beta^{4}\left[1+\alpha \delta\left(\xi-\xi_{0}\right)\right] Y(\xi)=0,
$$

with the following boundary conditions

$$
Y(0)=0, \quad Y^{\prime}(0)=0, \quad Y^{\prime \prime}(1)=0, \quad Y^{\prime \prime \prime}(1)=0,
$$

where $\beta^{4}=\omega^{2} \rho A L^{4} / E I, \alpha=m / \rho A L, \xi=x / L, \xi_{0}=x_{o} / L, L$ is the length of the cantilever, and $\omega$ is the vibration angular frequency. From the boundary conditions at the free end of the cantilever, the frequency equation can be obtained as

$$
F\left(\beta, \alpha, \xi_{0}\right)=a_{11} a_{22}-a_{12} a_{21}=0,
$$

where

$$
\begin{aligned}
& a_{11}=\psi_{1}^{\prime \prime}(1)+\alpha \beta^{4} \psi_{2}^{\prime \prime}\left(1-\xi_{0}\right) \psi_{1}\left(\xi_{0}\right), \\
& a_{12}=\psi_{2}^{\prime \prime}(1)+\alpha \beta^{4} \psi_{2}^{\prime \prime}\left(1-\xi_{0}\right) \psi_{2}\left(\xi_{0}\right), \\
& a_{21}=\psi_{1}^{\prime \prime \prime}(1)+\alpha \beta^{4} \psi_{2}^{\prime \prime \prime}\left(1-\xi_{0}\right) \psi_{1}\left(\xi_{0}\right), \\
& a_{22}=\psi_{2}^{\prime \prime \prime}(1)+\alpha \beta^{4} \psi_{2}^{\prime \prime \prime}\left(1-\xi_{0}\right) \psi_{2}\left(\xi_{0}\right), \\
& \psi_{1}(\xi)=(\cosh \beta \xi-\cos \beta \xi) / 2 \beta^{2},
\end{aligned}
$$
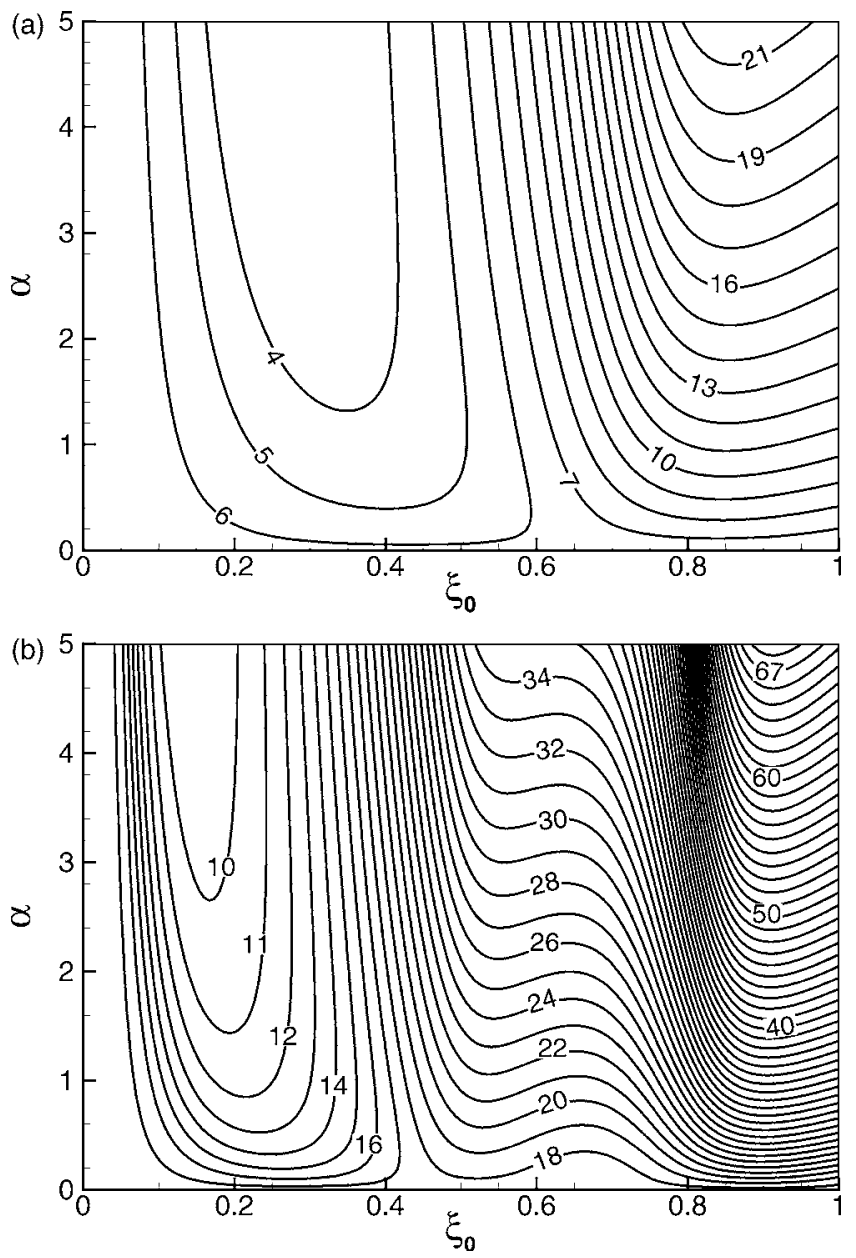

FIG. 2. Values of the concentrated mass $(\alpha)$ and its position $\left(\xi_{0}\right)$ which ensure that (a) $\omega_{2} / \omega_{1}$ or (b) $\omega_{3} / \omega_{1}$ are exactly integers. The integers are labeled on the corresponding curves.

$$
\psi_{2}(\xi)=(\sinh \beta \xi-\sin \beta \xi) / 2 \beta^{3},
$$

and the prime denotes differential with respect to $\xi$.

Provided the consecutive roots of Eq. (4) are denoted by $\beta_{i}(i=1,2,3, \cdots)$ for given $\alpha$ and $\xi_{0}$, the natural frequencies of the consecutive eigenmodes are given by $\omega_{i}$ $=\beta_{i}^{2} \sqrt{E I / \rho A L^{4}}$. Normalizing all natural frequencies with respect to the fundamental one $\left(\omega_{1}\right)$ gives $\omega_{i} / \omega_{1}=\left(\beta_{i} / \beta_{1}\right)^{2}$ $=f\left(\alpha, \xi_{0}\right)$. Consequently, two nondimensional parameters, $\left(\alpha, \xi_{0}\right)$, which represent the mass of the attached concentrated particle and its location, entirely determine the ratio $\omega_{i} / \omega_{1}$. By tuning $\left(\alpha, \xi_{0}\right)$, we can move resonance peaks of the higher-order eigenmodes in vibration spectrum back and forth with respect to that of the fundamental one. Therefore, carefully selected values of $\left(\alpha, \xi_{0}\right)$ match the natural frequency of a certain higher-order eigenmode to a chosen higher harmonic exactly. Such optimized values for $\left(\alpha, \xi_{0}\right)$, which enable the frequency response curve of tapping cantilevers to exhibit peaks at integer multiples of the fundamental natural frequency in order to coincide with higher harmonics, are given in Fig. 2, which shows the results for the second eigenmode and that for the third one respectively. The integer $n$ on each line in Fig. 2 labels each of the parameter curves that matches the natural frequency of a certain eigenmode to the $n$th harmonic $\left(\omega_{i} / \omega_{1}=n, i=2,3\right)$. When $\alpha$ varies from 0 to 5 , the 4 th-21st harmonics can be matched by the second eigenmode, while the 10th-68th can 
TABLE I. Values of $\left(\alpha, \xi_{0}\right)$ (for the case $\left.\alpha<0.5\right)$ which ensure that both $n_{2}=\omega_{2} / \omega_{1}$ and $n_{3}=\omega_{3} / \omega_{1}$ are integers.

\begin{tabular}{cccccccccccc}
\hline \hline$n_{2}$ & $n_{3}$ & $\alpha$ & $\xi_{0}$ & $n_{2}$ & $n_{3}$ & $\alpha$ & $\xi_{0}$ & $n_{2}$ & $n_{3}$ & $\alpha$ & $\xi_{0}$ \\
\hline 5 & 15 & 0.424 & 0.351 & 6 & 19 & 0.466 & 0.591 & 8 & 23 & 0.287 & 0.853 \\
5 & 16 & 0.396 & 0.386 & 7 & 18 & 0.341 & 0.679 & 8 & 24 & 0.310 & 0.906 \\
5 & 17 & 0.397 & 0.418 & 7 & 18 & 0.170 & 0.733 & 9 & 24 & 0.495 & 0.813 \\
5 & 18 & 0.428 & 0.448 & 7 & 19 & 0.119 & 0.799 & 9 & 25 & 0.484 & 0.831 \\
6 & 15 & 0.237 & 0.203 & 7 & 20 & 0.114 & 0.862 & 9 & 26 & 0.481 \\
6 & 16 & 0.101 & 0.281 & 8 & 20 & 0.341 & 0.768 & 9 & 27 & 0.493 \\
6 & 17 & 0.059 & 0.379 & 8 & 21 & 0.304 & 0.797 & & & 0.881 \\
6 & 18 & 0.194 & 0.586 & 8 & 22 & 0.289 & 0.823 & & & \\
\hline \hline
\end{tabular}

be matched by the third one. Thus, parameter curves in Fig. 2 provide a easy-to-use guide for the design of a harmonic cantilever. In fact, a carefully designed harmonic cantilever based on the guide can enhance a freely chosen higher-order harmonic of interest effectively.

A multiple harmonic cantilever, which allows the simultaneous excitation of the first three eigenmodes, can be achieved. When Fig. 2(b) is superimposed on top of Fig. 2(a), each of the intersection points between the curves in Fig. 2(a) and those in Fig. 2(b) define a multiple harmonic cantilever in the $\left(\alpha, \xi_{0}\right)$ parameter space. A typical frequency response curve for such a multiple harmonic cantilever is shown in Fig. 1. The second and third natural frequencies of such a cantilever coincide with two higher harmonics exactly (e.g., 5th and 15th harmonics in Fig. 1). Thus, the first three eigenmodes can be simultaneously excited with only one carefully positioned particle of specific mass, which results in greatly enhanced higher harmonic signals when the tip periodically taps on the sample surface at the fundamental natural frequency. As shown in Fig. 1, the 5th harmonic which may otherwise be heavily suppressed by the valley of the frequency response curve for an uniform cantilever is now significantly enhanced by the second resonance peak of the multiple harmonic cantilever. All intersection points are obtained for the case that the attached mass is not quite large $(\alpha<0.5)$ with the corresponding values of $\left(\alpha, \xi_{0}\right)$ listed in Table I. Such multiple harmonic cantilever can be quite useful in probing the tip-sample interaction. In addition to the increased image contrast based on the harmonic signals, two most important parameters of the pulse force of the tipsample interaction, the time duration and the strength, can be directly estimated from the two experimentally determined high-order harmonic components of the vibration amplitudes $A_{j}$ and $A_{k}$ enhanced by the second and the third eigenmodes $\left(\omega_{2}=j \omega_{1}, \omega_{3}=k \omega_{1}\right.$, both $j$ and $k$ are integers). When the pulselike interaction force is approximately described by a empirical function (e.g., cut cosine ${ }^{19}$ ), the harmonic enhancement characteristic of the multiple harmonic cantilever results in a greatly simplified analytical expression. Quickly accessing the duration and the strength of the tip-sample interaction provides a potential basis for the real-time quantitative characterization of material properties without affecting the simultaneous raster scanning of topography imaging and, thus, opens the possibility of developing a force spectroscopy not only to distinguish, but also to identify material composite.
In summary, we propose to tune the vibration spectrum characteristics of the cantilever probe of tapping-mode atomic force microscopy with an attached concentrated mass to enhance the higher harmonic vibration signals which contain the information on material properties of the sample surface. The analytical solution obtained based on the theory of Euler-Bernouli beams provides a comprehensive map to guide the choice of the two governing parameters, the mass of the attached particle and its position, to ensure achieving a harmonic cantilever in a flexible manner. The time duration and the strength of the pulselike tip-sample interaction force can be quickly accessed based on the significantly enhanced high-sensitive harmonic signals, which allows the combination of qualitatively extracting material properties with the simultaneous nanometer-resolution surface imaging capability.

Financial support from NSFC (Grant Nos. 10002020 and 10721202) and key project from Chinese Academy of Sciences (Nos. KJCX2-SW-L2 and KJCX2-YW-M04) are gratefully acknowledged.

${ }^{1}$ G. Binnig, C. Quate, and C. Gerber, Phys. Rev. Lett. 56, 930 (1986).

${ }^{2}$ Q. Zhong, D. Inniss, K. Kjoller, and V. Elings, Surf. Sci. Lett. 290, L688 (1993).

${ }^{3}$ R. Stark and W. Heckl, Rev. Sci. Instrum. 74, 5111 (2003).

${ }^{4}$ C. Lin and L. Segel, Mathematics Applied to Deterministic Problems in the Natural Sciences (Macmillan, New York, 1974).

${ }^{5}$ R. Hillenbrand, M. Stark, and R. Guckenberger, Appl. Phys. Lett. 76, 3478 (2000).

${ }^{6}$ M. Stark, R. Stark, W. Heckl, and R. Guckenberger, Appl. Phys. Lett. 77, 3293 (2000).

${ }^{7}$ O. Sahin and A. Atalar, Appl. Phys. Lett. 79, 4455 (2001).

${ }^{8}$ M. Stark, R. Stark, W. Heckl, and R. Guckenberger, Proc. Natl. Acad. Sci. U.S.A. 99, 8473 (2002).

${ }^{9}$ O. Sahin, C. Quate, O. Solgaard, and A. Atalar, Phys. Rev. B 69, 165416 (2004).

${ }^{10}$ J. Legleiter, M. Park, B. Cusick, and T. Kowalewski, Proc. Natl. Acad. Sci. U.S.A. 103, 4813 (2006).

${ }^{11}$ S. Crittenden, A. Raman, and R. Reifenberger, Phys. Rev. B 72, 235422 (2005).

${ }^{12}$ M. Balantekin and A. Atalar, Appl. Phys. Lett. 87, 243513 (2005).

${ }^{13}$ J. Preiner, J. Tang, V. Pastushenko, and P. Hinterdorfer, Phys. Rev. Lett. 99, 046102 (2007).

${ }^{14}$ J. Lozano and R. Garcia, Phys. Rev. Lett. 100, 076102 (2008).

${ }^{15}$ O. Sahin, G. Yaralioglu, R. Grow, S. Zappe, A. Atalar, C. Quate, and O. Solgaard, Sens. Actuators, A 114, 183 (2004).

${ }^{16}$ T. Rodriguez and R. Garcia, Appl. Phys. Lett. 84, 449 (2004).

${ }^{17}$ M. Balantekin and A. Atalar, Phys. Rev. B 71, 125416 (2005).

${ }^{18}$ O. Sahin, S. Magonov, C. Su, C. F. Quate, and O. Solgaard, Nat. Nanotechnol. 2, 507 (2007).

${ }^{19}$ R. Stark and W. Heckl, Surf. Sci. 457, 219 (2000). 